\title{
Correction to: Valorization of wastewater from food industry: moving to a circular bioeconomy
}

\author{
Carolina F. F. A. Costa $($ Catarina L. Amorim $(D)$ Anouk F. Duque $(1)$ \\ Maria A. M. Reis (iD) Paula M. L. Castro $(1)$
}

Published online: 27 December 2021

(C) Springer Nature B.V. 2021

\section{Correction to: Rev Environ Sci Biotechnol https://doi.org/10.1007/s11157-021-09600-1}

Following publication of the original article, the authors identified an error in the affiliations of the author. The correct version of authors affiliations are given below,

${ }^{1}$ Universidade Católica Portuguesa, CBQF - Centro de Biotecnologia e Química Fina - Laboratório Associado, Escola Superior de Biotecnologia, Porto, Portugal

The original article can be found online at https:// doi.org/10.1007/s11157-021-09600-1.

C. F. F. A. Costa · C. L. Amorim ( $₫)$ · P. M. L. Castro CBQF - Centro de Biotecnologia e Química Fina Laboratório Associado Escola Superior de Biotecnologia, Universidade Católica Portuguesa, Porto, Portugal

e-mail: camorim@ucp.pt

A. F. Duque - M. A. M. Reis

Associate Laboratory i4HB - Institute for Health and Bioeconomy, NOVA School of Science and Technology, Universidade NOVA de Lisboa, Caparica, Portugal

A. F. Duque - M. A. M. Reis

UCIBIO - Applied Molecular Biosciences Unit, Department of Chemistry, NOVA School of Science and Technology, Universidade NOVA de Lisboa, Caparica, Portugal
${ }^{2}$ Associate Laboratory i4HB - Institute for Health and Bioeconomy, NOVA School of Science and Technology, Universidade NOVA de Lisboa, Caparica, Portugal

${ }^{3}$ UCIBIO - Applied Molecular Biosciences Unit, Department of Chemistry, NOVA School of Science and Technology, Universidade NOVA de Lisboa, Caparica, Portugal

Publisher's Note Springer Nature remains neutral with regard to jurisdictional claims in published maps and institutional affiliations. 